\title{
MANIFESTACIONES OCULARES EN EL SÍNDROME DE PROTEUS
}

\section{OCULAR MANIFESTATIONS IN PROTEUS SYNDROME}

\author{
SÁNCHEZ-LÓPEZ M ${ }^{1}$, MARTÍNEZ-FERNÁNDEZ R ${ }^{1}$, SANTAMARÍA-CARRO A ${ }^{1}$
}

\section{RESUMEN}

Caso clínico: Las malformaciones congénitas deformantes son raras y tienen una etiología multifactorial. Presentamos las manifestaciones oculares de un caso clínico de Síndrome de Proteus. La retina mostraba una desorganización difusa, alteraciones pigmentarias e hipoplasia de nervio óptico. Otras alteraciones eran estrabismo y alta miopía.

Discusión: El Síndrome de Proteus es un complejo trastorno hamartomatoso caracterizado por un crecimiento local exagerado, tumores subcutáneos y diversas malformaciones óseas, cutáneas y/o vasculares. La incidencia de las malformaciones oculares en el Síndrome de Proteus es desconocida, precisando un examen craneofacial minucioso y un estudio sistemático ocular en estrecha relación multidisciplinaria para mejorar la asistencia de estos pacientes.

Palabras clave: Síndrome de Proteus, disgenesia retiniana, miopía, anomalías pigmentarias retinianas, hipoplasia de nervio óptico.

\begin{abstract}
Case report: Congenital disfiguring malformations are rare and usually have a multifactorial aetiology. Here we report on the ocular manifestations seen in a patient with Proteus syndrome. The retina showed retinal dysgenesia, retinal pigmentary abnormalities and optic nerve hypoplasia. Other abnormalities included strabismus and high myopia.

Discussion: Proteus syndrome is a complex hamartomatous disorder defined by local overgrowth, subcutaneous tumours and various bone, cutaneous and/or vascular anomalies. The incidence of ocular malformations in Proteus syndrome is unknown, however a meticulous cranio-facial examination and a systematic study of the eye is required to improve the medical care of these patients (Arch Soc Esp Oftalmol 2007; 82: 175-178).
\end{abstract}

Key words: Proteus syndrome, retinal dysgenesia, myopia, retinal pigmentary abnormalities, optic nerve hypoplasia.

\footnotetext{
Recibido: 2/1/06. Aceptado: 13/2/07.

Servicio de Oftalmología de Hospital de Cruces. Vizcaya. España.

1 Licenciado en Medicina.

Comunicación presentada en el Congreso Anual de la Sociedad Oftalmológica del Norte (San Sebastián 2005).

Correspondencia:

María Sánchez López

Hospital de Cruces

Plaza de Cruces, s/n

48903 Cruces/Barakaldo

España

E-mail:mariasanlo@terra.es
} 


\section{INTRODUCCIÓN}

El Síndrome de Proteus es un trastorno complejo y variable, descrito por Cohen y Hayden en 1979. Se presentan las manifestaciones oculares de un paciente afecto. Éstas son consecuencia de una malformación del desarrollo neurorretiniano. El síndrome fue acuñado científicamente en 1983 por Wiedemann et al como Síndrome de Proteus. En 1987, Happle postuló que es el resultado de un mosaicismo somático.

\section{CASO CLÍNICO}

Mujer nacida a término por parto vaginal. Primer hijo de padres no consanguíneos. Detección intraútero en ecografía de control de ventriculomegalia bilateral leve en la semana 21 de gestación que persiste en controles sucesivos. No se observan otras incidencias durante el embarazo. La puntuación en el test de Apgar fue 3/10, 4/10 y 8/10 a los 1, 10 y 20 minutos respectivamente, precisando intubación endotraqueal en la sala de partos. El peso al nacimiento fue 3.480 gramos, la talla $55 \mathrm{~cm}$ y perímetro craneal $39,5 \mathrm{~cm}$. Al nacimiento presenta rasgos dismórficos: fascies tosca, hipertelorismo, narinas antevertidas, microrretrognatia y asimetría frontal con prominencia izquierda. Hipotonía generalizada que progresa hacia hipertonía generalizada con reflejos vivos, clonus y episodios convulsivos generalizados. No hay seguimiento visual. Durante el período neonatal, se realiza un electroencefalograma presentando una discreta lentificación difusa. En la ecografia cerebral se observa ventriculomegalia asimétrica de predominio izquierdo y asimetría hemisférica a favor del lado izquierdo. En la tomografía computarizada craneal presenta una prominencia del sistema ventricular. En la resonancia nuclear magnética se observa displasia cortical con focos de heterotopía nodular (figs. 1 y 2).

El despistaje metabólico e infeccioso es normal. Cariotipo 46XX.

A los 7 meses de edad es remitida al servicio de oftalmología por esotropía del ojo derecho de $15^{\circ}$. Presenta dominancia de ojo izquierdo, nistagmus en abducción en ambos ojos. La retina muestra un aspecto distrófico con desorganización difusa y anomalías pigmentarias retinianas. Hipoplasia de nervio óptico (fig. 3).

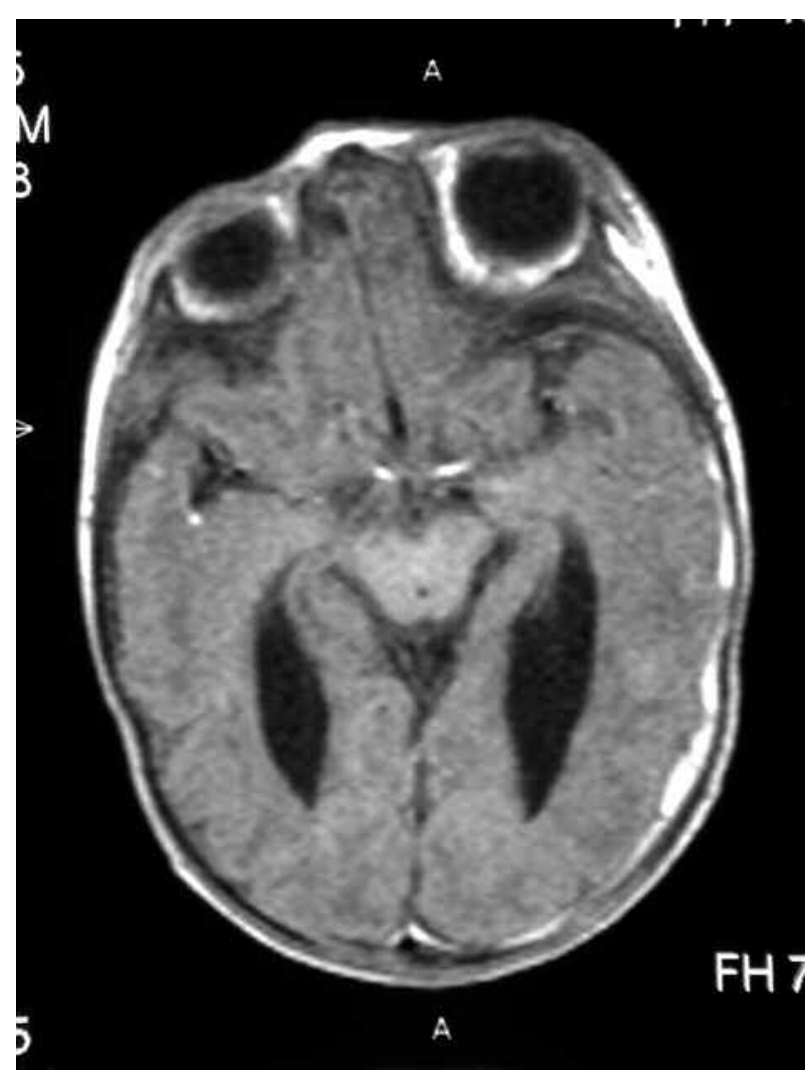

Fig. 1: Ventriculomegalia asimétrica de predominio izquierdo.

Presenta miopía de $-13,00$ dioptrías $\left(-2\right.$ a $90^{\circ}$ ) en ojo derecho y $-14,00$ dioptrías $\left(-2\right.$ a $\left.180^{\circ}\right)$ en ojo izquierdo. Se prescribe corrección óptica. Los potenciales evocados visuales resultan patológicos con un aumento del período de latencia. No se puede realizar un electrorretinograma por falta de colaboración. A los 14 meses, la gran hipertofia hemicraneal izquierda imposibilita portar gafas, se recetan lentes de contacto que son bien toleradas (fig. 4).

A los 10 meses, presenta un importante retraso psicomotor, hipertonía de extremidades e hipotonía cervicoaxial. No hay control cefálico ni sedestación. Mejora el contacto visual con mayor interacción con el ambiente y sonrisa social. Los controles electroencefalográficos muestran una asimetría interhemisférica del trazado, sin paroxismos irritativos. Presenta un progresivo aumento del hemicuerpo izquierdo, plagiocefalia y descenso de la órbita ipsilateral (fig. 5).

Aparición progresiva de deformidades: tibia vara, coxa valga y adelgazamiento femoral bilateral. A 


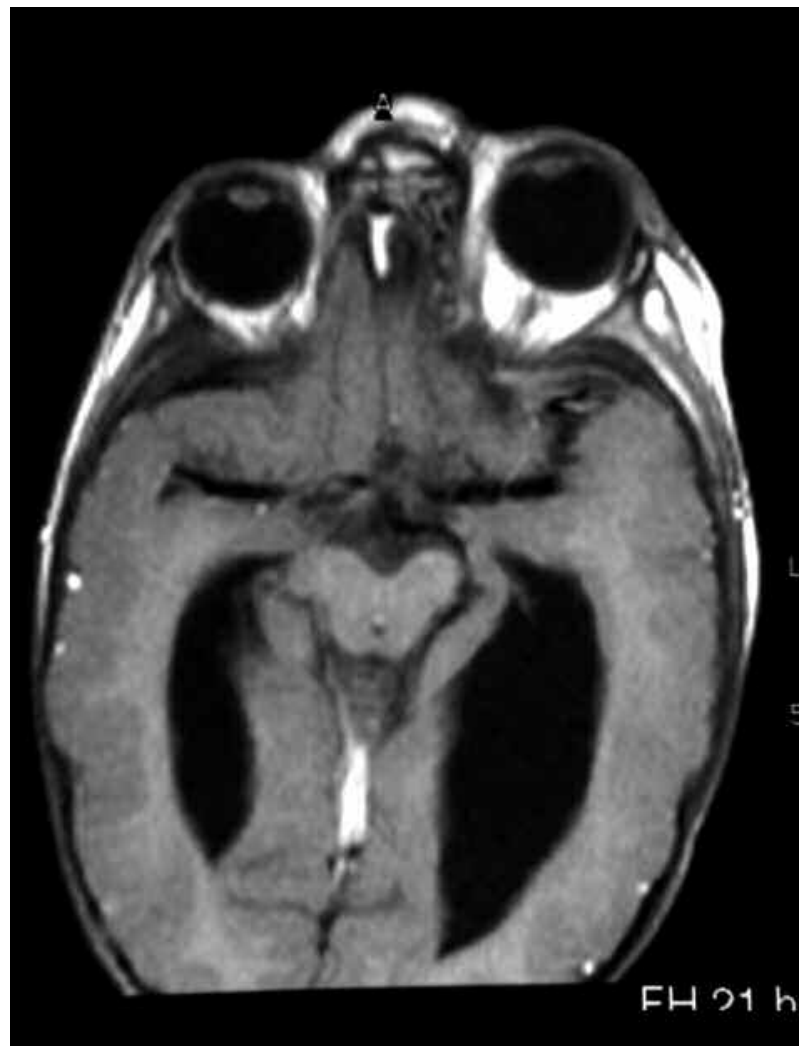

Fig. 2: Prominencia del sistema ventricular. Asimetría hemisférica a favor del lado izquierdo.

los 15 meses, presenta aspecto macrocefálico con afectación del macizo craneofacial. Nevus epidérmicos y hemangiomas dispersos con predominio en el lado izquierdo. Espasticidad como respuesta a

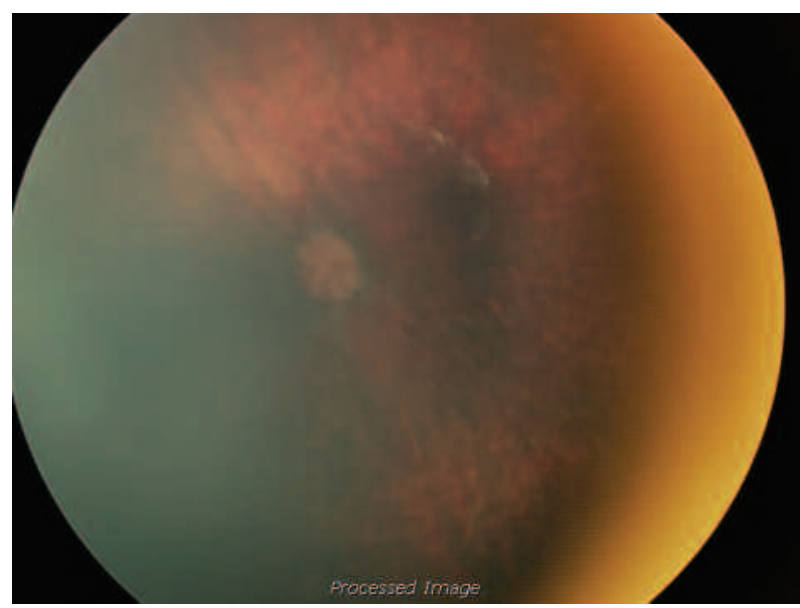

Fig. 3: Desorganización difusa y anomalías pigmentarias retinianas. Hipoplasia de nervio óptico.

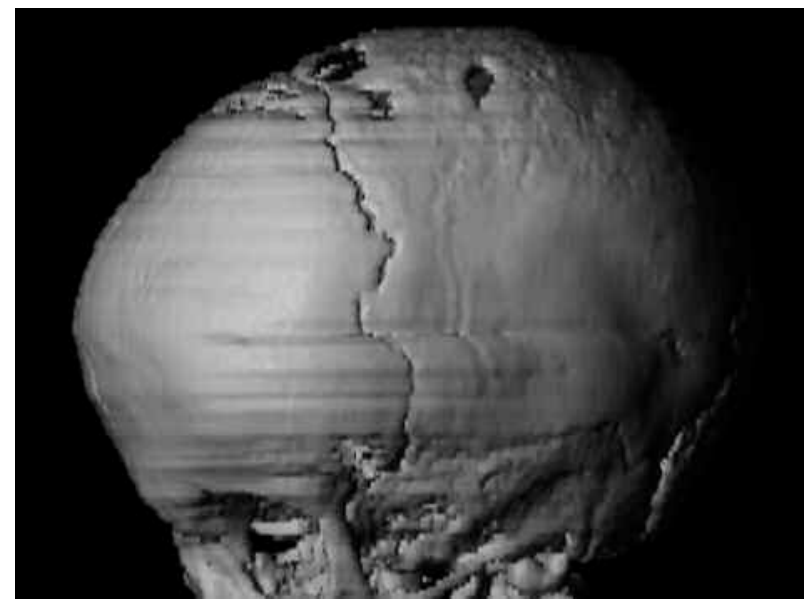

Fig. 4: Gran hipertrofia hemicraneal izquierda.

estímulos. A los 16 meses de edad, se realiza traqueostomía por laringotraqueomalacia. Fallece al sexto día del postoperatorio por neumonía.

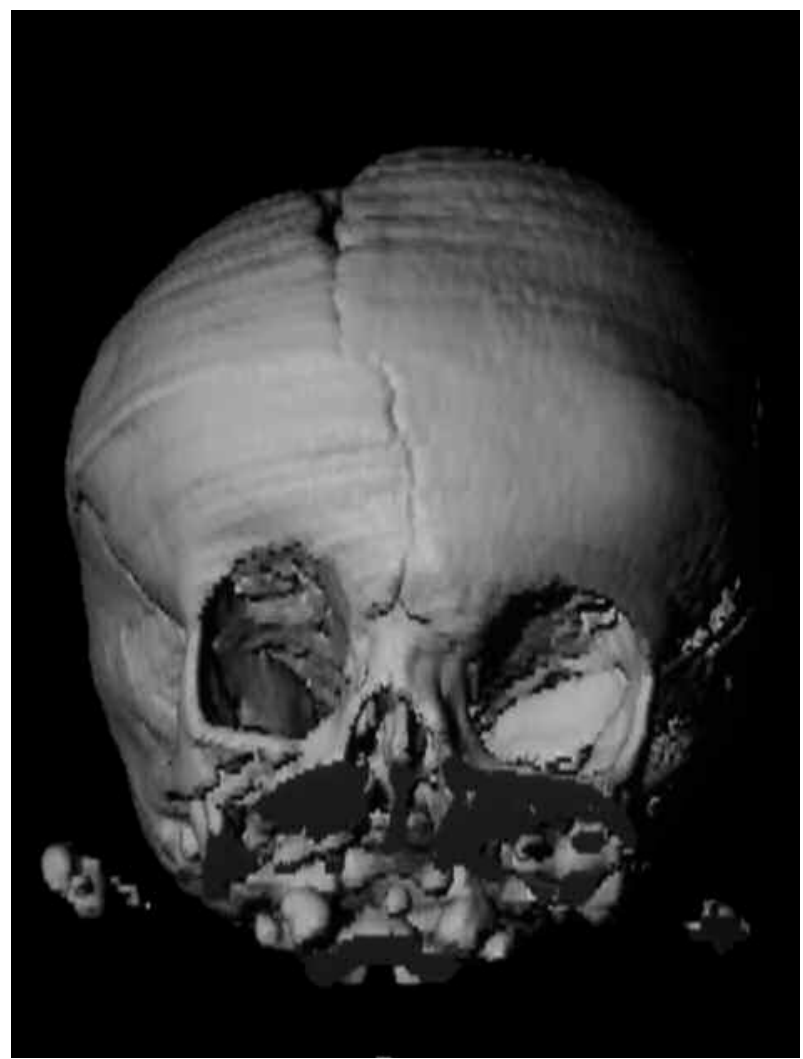

Fig. 5: Diez meses de edad. Plagiocefalia. Descenso de la órbita ipsilateral. 


\section{DISCUSIÓN}

El caso expuesto es un síndrome de Proteus de expresión precoz y severa, en el que se plantea una mala evolución global por convulsiones desde el período neonatal, laringotraqueomalacia,... finalmente, éxitus por neumonía, siendo ésta una de las causas de muerte ya recogidas por Cohen Jr (1). El trastorno es congénito, pero el diagnóstico suele ser tardío y se sospecha por el hipercrecimiento exagerado y progresivo de cualquier zona corporal, que suele cesar generalmente después de la pubertad. Se han ido describiendo casos de este síndrome en la literatura científica (más de 200 publicados) sin haber tenido gran repercusión en la literatura española en la que hay publicados, al menos, tres casos.

La tasa de diagnósticos erróneos es alta, ya que aunque se han publicado los criterios diagnósticos (2), éstos no han sido aplicados de manera consistente y fueron publicados después de que muchos casos fueran comunicados.

Se debe realizar un diagnóstico diferencial con otros cuadros (3), con fenotipos mosaicos neuroectodérmicos, tales como el Síndrome del nevus epidérmico, Lipomatosis encefalocraneocutánea, Síndrome de Delleman, Síndrome de Schimmelpenning, Síndrome de Goltz, Síndrome de Goldenhar,...

El pronóstico suele correlacionarse con la severidad de la afectación neurológica, de las deformidades esqueléticas, de la lipomatosis interna y con la aparición de neoplasias. De Becker et al (4) describen en el año 2000 las manifestaciones oculares del síndrome, siendo las más frecuentes el estrabismo y los tumores epibulbares. Asimismo, Sheard et al (5) describen una nueva presentación oftálmica que incluía, además de las ya descritas catarata y miopía, otras anomalías como la queratopatía en banda, anomalías de la estructura vítrea, hamartoma coriorretiniano asociado a desprendimiento seroso retiniano y hemorragia vítrea. En muchas ocasiones, no se realiza un estudio sistemático oftalmológico. Así mismo, en muchas publicaciones no se hace referencia a las manifestaciones oculares. Todo esto conlleva una infravaloración en la incidencia de manifestaciones oftalmológicas.

\section{BIBLIOGRAFÍA}

1. Cohen MM Jr. Causes of premature death in Proteus syndrome. Am J Med Genet 2001; 101: 1-3.

2. Turner JT, Cohen MM Jr, Biesecker LG. Reassessment of the Proteus syndrome literature: application of diagnostic criteria to published cases. Am J Med Genet A 2004; 130: 111-122.

3. Pinazo-Duran MD, Renal-Piqueras J, Guerri C, Stromland $K$. Optic nerve hipoplasia in fetal alcohol syndrome: an update. Eur J Ophthalmol 1997; 7: 262-270.

4. De Becker I, Gajda DJ, Gilbert-Barness E, Cohen MM Jr. Ocular manifestations in Proteus syndrome. Am J Med Genet 2000; 92: 350-352.

5. Sheard RM, Pope FM, Snead MP. A novel ophthalmic presentation of the Proteus syndrome. Ophthalmology 2002; 109: 1192-1195. 